\title{
La cocaína: consumo y consecuencias
}

\author{
Jesús del Bosque, ${ }^{1}$ Alba Fuentes Mairena, ${ }^{2}$ David Bruno Díaz, ${ }^{3}$ Mariana Espínola, ${ }^{4}$ Noé González García, ${ }^{5}$ \\ Arturo Loredo Abdalá, ${ }^{5}$ Ma. Elena Medina-Mora, ${ }^{6}$ Ricardo Nanni Alvarado, ${ }^{6}$ Guillermina Natera, ${ }^{6}$ \\ Oscar Prospero García, ${ }^{7}$ Ricardo Sánchez Huesca, ${ }^{3}$ Raúl Sansores, ${ }^{8}$ Tania Real, ${ }^{6}$ Juan Zinser, ${ }^{9}$ Lucía Vázquez ${ }^{6}$
}

Artículo original

\section{ANTECEDENTES}

El grupo que integra el Comité de Adicciones, ${ }^{*}$ ha analizado los principales problemas de adicciones de nuestro país con el fin de identificar campos para la colaboración interinstitucional y para hacer propuestas de políticas públicas. Este grupo ha publicado un documento sobre el abuso de bebidas con alcohol, y un trabajo más sobre el consumo de cannabis.

\section{INTRODUCCIÓN}

La cocaína deriva del nombre científico de la planta Erythroxylon coca: su aspecto es el de un polvo blanco, fino y cristalino. Fue aislada de las hojas de la planta por el químico alemán Friedrich Gaedcke, en 1885. Sin embargo, es a Albert Niemann a quién se le otorga el crédito de haberla aislado en 1859 como la conocemos. Los europeos la utilizaron un poco antes de que John Pemberton (1831-1887) inventara en 1886 el French Wine Coca, precursor de la CocaCola. En 1863, Angelo Mariani (1838-1914) inventó el famoso Vin Mariani. Esta bebida se elaboró con hojas de coca y vinos de Burdeos. Mariani, quien era químico, fabricó pastillas, elíxires e infusiones de cocaína. Todos estos productos se comercializaron con diversas indicaciones terapéuticas, particularmente para mejorar el estado de ánimo y reducir el cansancio. El papa León XIII era un entusiasta bebedor de Vin Mariani. Incluso es su efigie la que ostenta la etiqueta del vino desde $1997 .{ }^{1}$

Como una imitación del Vin Mariani, el doctor Pemberton inventó la Coca-Cola. En realidad, el doctor Pemberton era un adicto a la morfina y buscó refugio en la cocaína, ${ }^{1}$ que en ese entonces hasta Freud creía que podía reducir, controlar e incluso curar la dependencia al opio y sus derivados. ${ }^{2}$ Desde 1909, la Coca-Cola dejó de contener cocaína. ${ }^{3}$

Debido a que la cocaína es un estimulante poderoso, se empezó a observar que su consumo diario provocaba alteraciones en el sueño y pérdida del apetito, y si la persona no la consumía, podía entrar en un estado de desesperación. A raíz de ello se estableció la asociación entre el consumo y los efectos adversos. En Estados Unidos, estas conductas hicieron que se le prestara atención y se volviera una preocupación hasta que finalmente se prohibió su uso. En 1920, los legisladores se vieron obligados a incluir la cocaína en la lista de narcóticos prohibidos mediante la aprobación de la Ley de Drogas Peligrosas. Lamentablemente, el consumo de cocaína ya se había establecido entre la población y se había incorporado a la cultura. ${ }^{1}$

En México no se consideró un problema sino hasta la década de 1980, debido a las bajas prevalencias, y a que quienes la consumían pertenecían generalmente a clases socioeconómicas favorecidas, como escritores, intelectuales y artistas. A partir de la década de 1970 y, con mayor énfasis a partir de la década de 1990, se incrementó el consumo y se empezó a estudiar la forma de uso tanto de la cocaína fumada como de una nueva forma de presentación: el crack. Se estudiaron también los patrones de uso, el perfil de los nuevos usuarios y las diferencias por sexo. ${ }^{4} \mathrm{Su}$ uso se convierte en un problema alrededor del año 2000, que coincide con el cambio en las rutas internacionales de la zona del Caribe al corredor América central-México para llevar la droga a Estados Unidos. ${ }^{5}$

* Convocado por la Comisión Coordinadora de los Institutos Nacionales de Salud y Hospitales de Alta Especialidad.

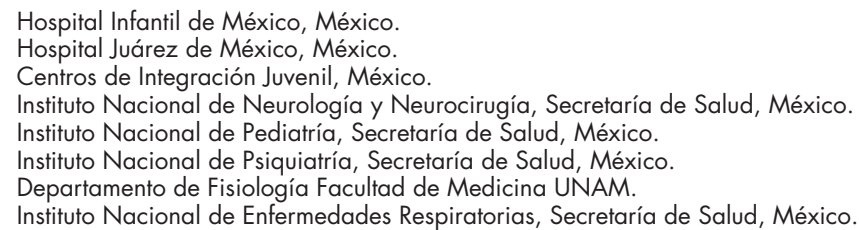




\section{NEUROBIOLOGÍA DE LOS EFECTOS DE LA COCAÍNA EN EL CEREBRO}

La cocaína se clasificó en el grupo I de la Convención Única de 1961 sobre Estupefacientes, enmendada por el protocolo de 1972. Aparece también en la lista de preparados de estupefacientes exceptuados de algunas disposiciones incluidas en la lista II de la Convención de 1961, a condición de que no contenga más de $0.1 \%$ de cocaína, calculado como cocaína base. ${ }^{6}$

Cuando se combina la cocaína con amoniaco o con bicarbonato de sodio y una solución acuosa, se obtiene el crack, unas piedrecillas con un alto potencial adictivo; su vía de administración es fumada. Otra presentación es la pasta base, que generalmente se consume más en la región andina y en otros países de América del Sur. Esta sustancia es el resultado de la extracción de la hoja de coca con keroseno y ácido sulfúrico y no con cocaína propiamente. Como el crack, la pasta base es una droga altamente peligrosa ya que su precio es bajo y su farmacocinética es de rápida absorción, pues se elimina aproximadamente en cinco minutos. Precisamente, las drogas con mayor capacidad adictiva son aquellas que se eliminan con mayor rapidez. El keroseno produce diversos efectos tóxicos, que incluyen la destrucción de la mielina. Éste es uno de los efectos más potentes y más dañinos del keroseno. Por su parte, el ácido sulfúrico produce también una amplia variedad de efectos tóxicos, que incluyen enfisema y cáncer de pulmón. ${ }^{7}$

Las vías de administración incluyen la oral 100-200 mg; la vía nasal, de $5 \times 30 \mathrm{mg}$; la inhalación de la cocaína, 60-250 $\mathrm{mg}$; de la pasta base de $60-250 \mathrm{mg}$ y el crack, en que no está clara la dosis. La cocaína se metaboliza rápidamente $(90$ min) a benzoilecgonina y a ecgonina metil ester, los cuales son inactivos. Se ha observado que se elimina un máximo del $10 \%$ de la cocaína sin cambios por orina. ${ }^{7}$

El usuario utiliza generalmente cocaína por el efecto estimulante y la sensación de autoconfianza. Es clasificada por algunos como una "ego droga". La persona puede experimentar la sensación de locuacidad y de rapidez de pensamiento, que con el paso del tiempo se puede tornar en irritabilidad y agresividad. Pero el efecto termina pronto, en 30 minutos, máximo 90, y el usuario puede experimentar sensación de pérdida de autoconfianza, miedo y ansiedad, que lo hace exigir una nueva dosis. Sin embargo, si el sujeto consume al mismo tiempo alcohol, se forma un compuesto llamado cocaetileno, que tiene una vida media mayor (150 $\mathrm{min})$, pero que al final tiene el mismo efecto displacentero. ${ }^{3}$

Los mecanismos neurobiológicos del consumo de cocaína incluyen la facilitación de la biodisponibilidad de dopamina en el sistema de la motivación-recompensa. La cocaína interfiere con el transportador de serotonina (SERT), el de dopamina (DAT) y el de norepinefrina (NET), en este orden de potencia. ${ }^{7}$

Estos transportadores son proteínas que se expresan en las terminales de estos neurotransmisores y están encargados de transportar la serotonina, la dopamina y la norepinefrina de regreso al interior de sus respectivas terminales sinápticas. ${ }^{8}$ Esto quiere decir que la recaptura de los neurotransmisores es uno de los mecanismos que usan las neuronas para terminar con la acción de dichos neurotransmisores. Es claro entonces que, si no se recaptura la dopamina, ésta permanece en el espacio sináptico, con lo que aumenta el tiempo de activación de los receptores dopaminérgicos. El área tegmental ventral (ATV) es la región cerebral que sintetiza y libera dopamina. Sus sitios blanco son el núcleo accumbens, la amígdala y la corteza prefrontal, entre otros. ${ }^{9}$ Así, el aumento de dopamina por interferencia con el DAT aumenta la dopamina en estos sitios blanco. Debido a que se ha observado en el núcleo accumbens que la dopamina se acopla a sus receptores D1 y D2, se ha determinado que su acción sobre los D2 facilita la instalación de la dependencia. Sin embargo, con la administración crónica, los D2 reducen su expresión, por lo que no se cree que participen en el mantenimiento de la adicción. Esto se ha observado usando la técnica de tomografía con emisión de positrones (PET), aunque no sabemos si los D2 reducen su expresión a consecuencia del uso de cocaína o si porque los pacientes lo expresaban poco, se volvieron adictos a la misma. Sin embargo, hay estudios con voluntarios (no adictos a cocaína), a los cuales se les administró metilfenidato. Aquellos a los que se les había detectado por PET que tenían menor expresión de D2 reportaron sentir placer con los efectos de este fármaco, no así los que tenían una alta expresión del D2, quienes reportaron displacer.

Estudios con ratas a las que se les sobreexpresa el D2, beben menos alcohol que las controles. Sin embargo, como beben, aunque sea poco, va reduciéndose la expresión del D2, por lo que las ratas van bebiendo más alcohol. Asimismo, se ha observado que los machos dominantes de una tropa de monos tienen el D2 más alto que los subordinados. Cuando se les somete a la autoadministración de cocaína, el subordinado ingiere más que el dominante. En algunos monos, a los cuales se les ha administrado cocaína por semanas o meses, y luego se les deja abstinentes por tres semanas o hasta un año, los D2 recuperan su expresión basal. ${ }^{10}$

Es decir, la dopamina y sus receptores, en particular el D2, así como el transportador de dopamina, son sitios importantes para la instalación y mantenimiento del consumo de cocaína. En la recaída, se ha estudiado ampliamente y se ha demostrado que el glutamato cumple una función muy importante. Los antagonistas del receptor AMPA glutamatérgico aplicados al núcleo accumbens, al área tegmental ventral y a la corteza prefrontal previenen la recaída en la autoadministración de cocaína. ${ }^{11}$ De forma contraria, la administración de agonistas en estas regiones cerebrales facilita la recaída.

Dentro de los sistemas ejecutivos encontramos la corteza prefrontal, los núcleos: globo pálido interno (GPi) y la habénula. La tercera circunvolución frontal derecha ha estado muy involucrada en la inhibición de la expresión de la conducta. Esta corteza activa el núcleo subtalámico y éste 
aumenta la actividad del globo pálido interno, con ello se inhibe el tálamo y se reduce la expresión de la conducta. ${ }^{12}$ Por su parte, la habénula activa las neuronas inhibidoras del núcleo rostromedial tegmental y éste inhibe a su vez la ATV. ${ }^{13}$ La falla en cualquiera de estos dos sistemas facilita la impulsividad del sujeto y su consumo de cocaína.

\section{EPIDEMIOLOGÍA DEL CONSUMO DE COCAÍNA EN POBLACIÓN MEXICANA}

La última Encuesta Nacional de Adicciones ${ }^{14}$ muestra que, en un periodo de nueve años (2002-2011), el consumo de drogas ilegales alguna vez en la vida aumentó en la población de 12 a 65 años, de $0.8 \%$ a 1.8\%. El consumo de cocaína ha mostrado variaciones en las preferencias de la población a finales de la década de 1980 e inicios de la de 1990, que es cuando alcanza mayor presencia en el mercado nacional. Posteriormente, se empareja con el nivel de consumo de otras sustancias para incrementar de nuevo su consumo a principios del año 2000.

Mientras que el consumo de otras drogas se duplicó entre 1988 y 2008, el de la cocaína aumentó en más de siete veces, de 0.33 a 2.60 en la población urbana entre 12 y 65 años. ${ }^{15,16}$

Actualmente, la cocaína (incluido el crack), seguida de la mariguana, es una de las sustancias preferidas por la población y su consumo alguna vez en la vida se incrementó de 2002 a 2011, de 0.3 a 0.5\%. Este orden de preferencia es igual tanto para hombres como para mujeres, con una razón de 2 a 1, respectivamente. Entre el grupo de mujeres, el consumo de cocaína y crack es mayor entre el grupo de edad de las más jóvenes de 12 a 25 años. Se observa que las generaciones actuales presentan mayor accesibilidad a las drogas, mayor consumo y mayor probabilidad de progresar del abuso a la dependencia que las generaciones anteriores. ${ }^{16}$

En relación con otras drogas se documentó que aquellos que consumen tabaco y alcohol antes de los 18 años in-

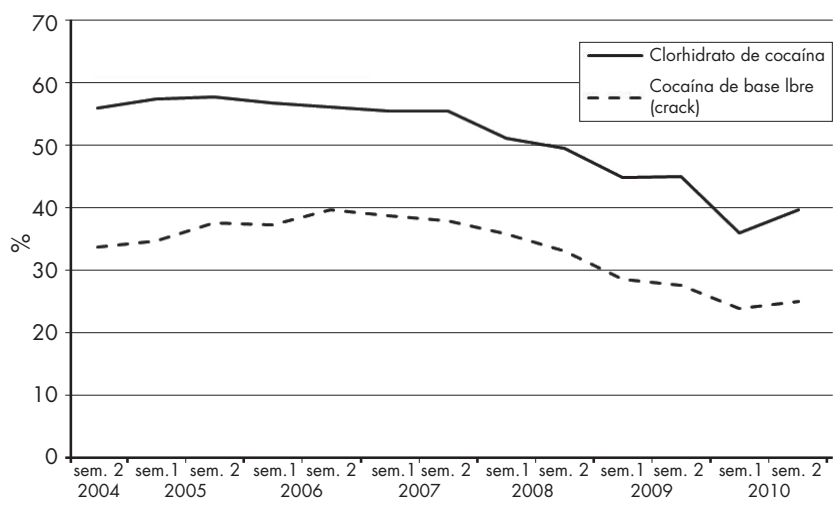

Figura 1. Uso de cocaína y crack alguna vez en la vida en usuarios de drogas solicitantes de tratamiento en $\mathrm{ClJ}$ del segundo semestre de 2004 al segundo semestre de 2010. crementan la probabilidad de usar otras drogas. En el caso de la cocaína, $7.6 \%$ de los usuarios experimentaron tempranamente con tabaco y $3.8 \%$ lo hizo con alcohol.

El consumo de cocaína por regiones en el país indica que en el sur el consumo es ligeramente más alto $(0.6 \%)$ que en la región centro $(0.2 \%){ }^{16}$

Por su parte, el Sistema de Reporte de Información en Drogas, ${ }^{17}$ en su último informe de la Ciudad de México, muestra estadísticas de 45 instituciones de atención a la salud y procuración de justicia, donde se captaron 1261 casos de los cuales 429 (34\%) fueron usuarios de cocaína; 35.4\% fueron hombres y $27.5 \%$ mujeres. En la categoría "alguna vez en la vida", 34\% utilizó cocaína y en la categoría "último mes" la consumió el 24\%. Al preguntar por sustancia de inicio, 8.2\% de los hombres lo hicieron con esta sustancia. Los usuarios de esta sustancia iniciaron antes de los 11 años, con $0.7 \%$. El grupo más afectado es el de los 15 a 19 años de edad, en $45.9 \%$.

La Encuesta de Estudiantes del D.F. ${ }^{18}$ indicó que de 2006 a 2009 se presentó un incremento en la prevalencia de consumo, de 17.8 a $21.5 \%$; este consumo es mayor en los hombres que en las mujeres (7.9 vs $6.1 \%$ ), y mayor entre adolescentes de bachillerato (28.5\%) en comparación con los de secundaria (16.1\%). Los estudiantes prefirieron en tercer lugar el consumo de cocaína $(3.5 \%)$ por debajo de la mariguana y los inhalables. Sin embargo, durante las mediciones que se realizan cada tres años se observa que el consumo de cocaína se mantiene estable con respecto a 2006. Un dato importante es que $75 \%$ de los estudiantes consideran peligroso el consumo de cocaína, lo que podría influir como un mecanismo preventivo.

Otra fuente de información se obtiene por medio de los usuarios de drogas que solicitaron tratamiento en Centros de Integración Juvenil (CIJ), ${ }^{19}$ donde se observó un incremento del porcentaje de uso alguna vez en la vida, de $12.2 \%$ en 1990 a $71.4 \%$ en 2000 . Hasta 2007, el consumo de cocaína alguna vez en la vida se mantuvo relativamente estable, pero en el periodo de 2007 a 2010 se mostró nuevamente una tendencia a la baja para situarse -en términos de uso alguna vez en la vida- en niveles cercanos o incluso inferiores a $50 \%$ (48.7\% en el segundo semestre de 2010)(figura 1). Tanto el consumo de cocaína como el de cocaína de base libre o crack presentan separadamente tendencias decrecientes para llegar a localizarse, en el segundo semestre de 2010, en 39.6 y $24.8 \%$, respectivamente.

Se reportan altos porcentajes de consumo de cocaína en prácticamente todos los centros de atención de todo el territorio nacional, mientras que el crack se reporta con mayor frecuencia por usuarios de sustancias atendidos en la región nororiental, del centro y del sur del país (figura 2).

La comparación de casos que señalaron la cocaína o el crack como droga de mayor impacto* en los CIJ muestra la

* Aquella cuya utilización les ha reportado mayores problemas y ha motivado la búsqueda de tratamiento. 


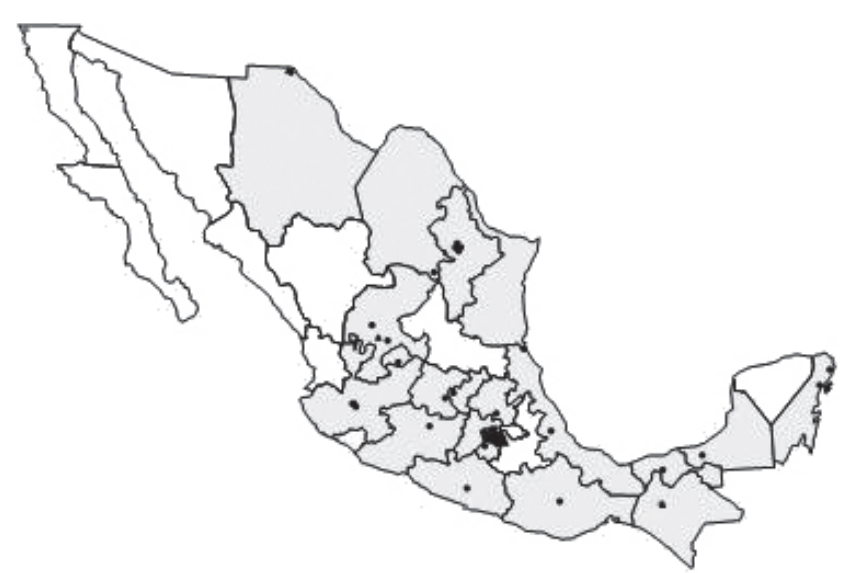

Figura 2. Unidades de $\mathrm{CIJ}$ en las que la población atendida reporta porcentajes de uso de crack alguna vez en la vida superiores a la media nacional (24.8\%). Segundo semestre de 2010.

existencia de algunas diferencias significativas. En primer lugar, los usuarios de crack como sustancia de mayor impacto presentan una proporción significativamente mayor de hombres y menor de mujeres ( 89.3 y $10.7 \%$, respectivamente) que los usuarios de cocaína (84.1 y 15.9\%); asimismo, comprenden un mayor porcentaje de desempleados (32.3 vs. 22.8\%) y un menor porcentaje de estudiantes activos (6.9 vs. 15.1\%).

De igual modo, quienes señalan el crack como droga de mayor impacto refieren haber utilizado un mayor número de drogas ilícitas alguna vez en la vida (3.37 en promedio [DE: 1.8] vs. 2.76 [DE: 1.9] que quienes reportaron la cocaína como droga de mayor impacto). Refieren también un mayor índice de consumo de tabaco (90.6 vs. 85.9\% de los usuarios de cocaína), inhalables (36.7 vs. 18.6\%), Rohypnol (7.1 vs. $4.1 \%$ ) y sustancias alucinógenas ( 8.0 vs. $4.7 \%$ ). Por su parte, quienes señalan como droga de mayor impacto la cocaína presentan un mayor porcentaje de uso alguna vez en la vida de estimulantes (22.9 vs. $14.4 \%)$ y, en particular, de metanfetaminas (11.7 vs. 5.6\%). Por último, los usuarios de crack como droga de mayor impacto presentan una mayor frecuencia de uso de drogas ilícitas en el último mes; así, 42.7 vs. $29.2 \%$ refieren haberlas utilizado diariamente. ${ }^{20}$

\section{FACTORES DE RIESGO DEL CONSUMO DE COCAÍNA}

De acuerdo con la investigación, el consumo experimental de cocaína se asocia con factores comunes al uso de sustancias en general, como: una alta accesibilidad, una baja percepción de riesgo, la presión de pares, un pobre control conductual y una baja adherencia escolar. Sin embargo, estudios desarrollados en CIJ han descrito que el usuario de cocaína mantiene un concepto negativo de la familia (a la que percibe como agresiva y no confiable), de las relaciones interpersonales (percibiendo a los amigos como no confia- bles) y de sí mismo (percibiéndose como agresivo e insatisfecho, entre otros aspectos). ${ }^{21}$ Como parte del grupo de consumidores de estimulantes mayores, los usuarios de cocaína acusan la existencia de trastornos del control de impulsos, baja autoestima y depresión (con sentimientos de fracaso, decepción, castigo, culpa y autocrítica, principalmente). Asimismo, reportan estar expuestos a relaciones violentas en la familia, con antecedentes de violencia física, emocional y, en algunos casos, sexual, factores que, a su vez, se asocian con actividades delictivas y conductas violentas reportadas por los propios usuarios. ${ }^{19,22}$ Finalmente, se registró una insatisfacción en las necesidades básicas de seguridad (tranquilidad, seguridad emocional y confianza, entre otras).

\section{COCAÍNA Y EFECTOS EN LA SALUD}

Además de los efectos a nivel del Sistema Nervioso que produce la cocaína, se han observado otros en el resto del organismo. Dentro de los inmediatos encontramos: vasoconstricción, midriasis, hipertermia, taquicardia e hipertensión. Los efectos derivados de la euforia, principalmente durante los primeros 30 minutos son la hiperestimulación, la sensación de menos cansancio y un estado de mayor alerta mental. Otros efectos frecuentes a mediano y largo plazo son: arritmias, infarto miocárdico, dolor torácico, insuficiencia respiratoria, afecciones vasculares cerebrales, convulsiones, cefalea, náusea, dolor abdominal, anorexia y desnutrición.

Existen también efectos específicos dependiendo de la vía de administración. La vía intranasal puede ocasionar: epistaxis, anosmia, perforación del tabique, disfonía y disfunción en la deglución. La vía oral puede ocasionar isquemia intestinal y la inyectada: alergias, VIH, hepatitis y otras infecciones.

Los efectos dependen de la sensibilidad individual y de la dosis, la cual se relaciona con la vía de administración y la pureza del producto, que por lo menos en $40 \%$ de los casos es impuro, encontrándose adulterada con talco, maicena o azúcar. También es adulterada con procaína o con anfetaminas. ${ }^{23}$

Un factor que puede potenciar el efecto biológico de la cocaína es la fracción libre en sangre. El $90 \%$ se une a la albúmina, de tal manera que cuando ésta disminuye, el efecto con la misma dosis puede ser mayor. Como consecuencia, de la anorexia y las frecuentes transgresiones dietéticas experimentadas al consumir cocaína, la hipoalbuminemia puede ser uno de los efectos que a su vez agrave otros.

A diferencia de otras drogas, dentro del círculo vicioso del consumidor de cocaína son evidentes los efectos agudos y crónicos en la salud. La hiperestimulación y la sensación de mayor energía pueden elevar el umbral de tolerancia a otros síntomas, como los cardiovasculares, respiratorios y dolorosos, que de no atenderse pueden llevar a consecuen-

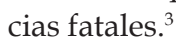




\section{COCAÍNA Y EMBARAZO}

Tras el aumento en la prevalencia del uso de cocaína en la población general, debido a su creciente disponibilidad ${ }^{24} \mathrm{y}$ su reflejo en la tendencia de consumo en mujeres en edad reproductiva, se estima que el número de mujeres embarazadas que la consumen también se ha incrementado. ${ }^{25}$

Aunque resulta complejo valorar la prevalencia exacta del consumo de cocaína durante el embarazo debido a la variabilidad de poblaciones y metodologías utilizadas, algunos estudios realizados alrededor del mundo establecen una tasa entre 1.8 y $18 \%,{ }^{26-28}$ con lo que se amplía el carácter nocivo que surte la exposición a la cocaína durante el embarazo en el bienestar materno, fetal y neonatal.

Basados en los principios del modelo teratológico-neuroconductual, numerosos estudios han descrito el impacto que tiene la exposición prenatal a la cocaína en la salud, el Sistema Nervioso Central, la conducta y el desarrollo del niño, ${ }^{29}$ asociado a la constitución genética, el medio ambiente (perinatal y post natal), dosis y etapa de desarrollo al momento de la exposición.

Es importante mencionar que los cambios fisiológicos asociados al embarazo tienen un efecto en la absorción, la distribución, el metabolismo y la eliminación ${ }^{30}$ de la cocaína, con lo que se incrementa su efecto nocivo en la madre y en el feto.

La actividad de la colinesterasa plasmática, que metaboliza la cocaína a ecgonina y benzoilecgonina, se reduce en mujeres embarazadas, disminuyendo la velocidad en que ésta se metaboliza en compuestos inactivos, con lo que potencia así los efectos nocivos de la misma en la madre y el feto. ${ }^{24}$ Las mujeres embarazadas metabolizan la cocaína a norcaine activo en un grado mucho mayor; de este modo, madre e hijo están expuestos a esta alta concentración del metabolito activo. ${ }^{31,32}$

Debido a las propiedades lipofílicas de la cocaína, ésta atraviesa rápidamente la barrera placentaria por difusión simple, con lo que expone al feto a una concentración elevada de la misma. ${ }^{33}$

La respuesta sistémica a la cocaína entraña efectos cardiovasculares (vasoconstricción, hipertensión y taquicardia), que favorecen: contracción de las arterias umbilicales, insuficiencia placentaria y restricción del crecimiento uterino (RCIU). ${ }^{34}$

Su carácter hipertensivo y el aumento en la contractibilidad uterina por el incremento en los niveles de norepinefrina predispone el desprendimiento placentario;, ${ }^{35,36} \mathrm{el}$ incremento en los niveles plasmáticos de oxitocina mostrado en modelos animales sugiere y explica el trabajo de parto y parto prematuro,; 37,38 además, su consumo en el primer trimestre incrementa el riesgo de aborto espontáneo. ${ }^{39}$

Las malformaciones congénitas ocurren entre 7 y $17 \%$ de los recién nacidos expuestos a cocaína (siendo importante recordar el consumo concomitante de alcohol en el 6090\% de los consumidores). Al ser lipofílica y de peso mole- cular relativamente bajo, le es posible atravesar la placenta y la barrera hematoencefálica, lo que da lugar a taquicardia fetal, disminución de la variabilidad del latido, falta de aceleración e hipertensión (trazo cardiotocográfico anormal). El síndrome de abstinencia se presenta entre $10-40 \%$ de los neonatos expuestos a cocaína. ${ }^{40,41}$

Por el efecto sobre los sistemas neurotransmisores monoaminérgicos (dopamina, norepinefrina, epinefrina y serotonina), se ve afectado el desarrollo neurológico ${ }^{42-44}$ alterando a largo plazo el circuito del aprendizaje, atención, inhibición ${ }^{45}$ y lenguaje ${ }^{46}$ también se han reportado alteraciones en el crecimiento (altura, peso, perímetro encefálico). ${ }^{47}$

Dichas complicaciones se relacionan con la dosis, el tiempo de consumo y la etapa gestacional; además, reflejan en ocasiones el impacto de múltiples exposiciones que combinan el consumo de cocaína con otras sustancias (alcohol, tabaco, cannabis y heroína) para actuar de forma sinérgica y agravar los efectos adversos en la madre y en el feto.

Dentro del contexto del abuso de sustancias en mujeres embarazadas se han descrito otras características que implican altos niveles de estrés, ausencia de cuidado prenatal, conductas de riesgo y exposición a la violencia; en usuarias de cocaína intravenosa existe una mayor prevalencia de enfermedades de transmisión sexual (VIH y hepatitis C).

\section{COMORBILIDAD PSIQUIÁTRICA EN CONSUMIDORES DE COCAÍNA}

El diagnóstico dual (coexistencia de psicopatología y consumo de sustancias psicoactivas) se sustenta en bases neurobiológicas. Se ha descrito una disfunción de los sistemas noradrenérgicos, serotoninérgicos y dopaminérgicos, localizados en el área septohipocámpica y en la amígdala, ${ }^{48}$ dichos sistemas están involucrados en la inhibición conductual.

Estos cambios reducen la capacidad del control de los impulsos y de la percepción de los fenómenos de recompensa, con lo que se incrementa la vulnerabilidad para el desarrollo de trastornos por uso de cocaína..$^{49}$ En el Trastorno Depresivo Mayor se ha podido evidenciar un déficit dopaminérgico, que es modificado por el sujeto al consumir la cocaína incrementando las concentraciones de dopamina en el núcleo estriado, así como la sensibilidad a los agonistas dopaminérgicos directos o indirectos, de forma secundaria a un incremento de la función en el núcleo accumbens.

El Factor Liberador de Corticotrofina (CRF) es un péptido hipotalámico distribuido en áreas límbicas y en núcleos del tronco cerebral que al liberar la corticotropina regula la respuesta a diferentes situaciones de estrés implicadas en los trastornos depresivos y ansiosos.

Por otro lado, durante el síndrome de abstinencia a sustancias psicoactivas hay un incremento de la neurotransmisión de CRF, hecho que parece indicar la existencia de una alteración neurobiológica común entre la depresión y 
la dependencia a sustancias. Así mismo, en pacientes deprimidos se ha podido detectar una disminución en el líquido cefalorraquídeo de las concentraciones de Neuropéptido Y (NPY), existiendo tasas opuestas entre este neuropéptido y el CRF. ${ }^{50}$ Durante la abstinencia a cocaína se produce una disminución de la neurotransmisión mediada por NPY. Además, las personas vulnerables que consumen cocaína pueden desarrollar un trastorno psicótico secundario a su uso por un fenómeno de sensibilización progresiva que se manifiesta clínicamente, en la mayoría de los casos, como episodios psicóticos transitorios.

Esta sensibilización puede atribuirse a un desequilibrio entre los receptores dopaminérgicos D3 y los receptores D1 y D2, pues se ha observado una mayor afinidad de la cocaína por el receptor D3, responsable de un desarrollo más rápido de tolerancia. ${ }^{51}$ Los trastornos de la personalidad con frecuencia asociados al uso de cocaína son: el trastorno antisocial y el trastorno límite. Así, mientras diversos autores postulan que la agresividad, la hiperactividad y la impulsividad son rasgos característicos de las personas que desarrollan dependencia a cocaína, otros defienden que el efecto comportamental secundario al consumo compulsivo de sustancias de abuso puede determinar que los diagnósticos de trastorno antisocial y límite se incrementen en un 19.2 y $11.2 \%$, respectivamente. De modo independiente a uno u otro postulado, un estudio reciente, realizado con una muestra de 3360 parejas de gemelos varones incluidos en un registro específico de veteranos de la guerra de Vietnam (Vietnam Era Twin Registry), confirma la estrecha relación existente, desde la perspectiva genética, entre el trastorno antisocial de la personalidad y la dependencia a cocaína. ${ }^{52}$

Diversos estudios han confirmado la elevada prevalencia de antecedentes de trastorno por déficit de atención con hiperactividad (TDAH) en sujetos con dependencia y/o abuso de cocaína. La prevalencia de trastornos por uso de cocaína en pacientes con diagnóstico de trastorno por déficit de atención también es muy elevada. Se ha descrito un hipofuncionamiento dopaminérgico en pacientes con TDAH, en los que se ha podido demostrar una reducción de la dopamina extracelular, así como un incremento en la densidad de los transportadores de dopamina y en las descargas neuronales incontroladas. ${ }^{53}$

\section{VIOLENCIA Y SU RELACIÓN CON EL CONSUMO DE SUSTANCIAS}

Algunos estudios han encontrado que el abuso de drogas es un factor para cometer delitos como el homicidio y el robo; en este sentido, la evidencia sugiere que existe una asociación de mayores índices de criminalidad con mayor abuso de drogas. Pero al mismo tiempo, se sabe que no todos aquellos que abusan de las drogas se tornan violentos o cometen actos criminales. $^{54}$
Cabe destacar que la violencia está presente en las sociedades no sólo por el tráfico y el uso de drogas, pues también intervienen elementos culturales y ambientales, y este fenómeno tiene impacto a nivel global y microsocial. En general, la industria del mercado ilegal de drogas desestabiliza la economía de los países, así como la de la sociedad civil, como resultado del incremento del crimen, la corrupción del sistema legal y político, el abuso de drogas y la pérdida de la cohesión social.

El incremento de actos criminales genera gastos específicos por uso de servicios médicos, funerarios, de seguridad y tratamiento, especialmente si la sociedad ha estado expuesta por periodos prolongados.

Otro fenómeno relacionado con el crimen es lo atractivo que puede resultar para los jóvenes involucrarse en el tráfico de drogas, por las ganancias que éste implica, motivo por el cual abandonan la escuela. El crimen y la violencia, relacionados con el abuso de drogas, tienen un impacto en diferentes niveles de la sociedad, que van desde problemas internacionales relacionados con el tráfico y con las actividades de organizaciones criminales, a los perpetrados contra los individuos que abusan de las drogas y los inocentes que quedan atrapados en fuegos cruzados, o aquellos crímenes relacionados con la necesidad que tienen los usuarios y las personas dependientes de obtener dinero o recursos para conseguir las drogas. ${ }^{54,55}$

Explicar la relación entre drogas y crimen es complicado, pues requiere integrar una visión que se centre en el individuo (aspectos físicos, psicológicos e incluso factores psiquiátricos y farmacológicos), en la visión social y cultural (reparto de los bienes, diferencias socioeconómicas), todos ellos factores, que, combinados, tienen un impacto.

En el caso de la cocaína se ha encontrado que el abuso se asocia con un incremento en la probabilidad de perpetrar un crimen violento, y específicamente, en el caso del crack, se ha documentado el efecto psicofarmacológico de éste y su relación con el aumento en la portación de armas. ${ }^{56}$

También se ha documentado que existe mayor riesgo de victimización en las personas que consumen drogas, ya que esto los vulnera de manera temporal o permanente por la incapacidad que sufren para responder e interpretar situaciones peligrosas.

\section{CONCLUSIONES}

Los datos presentados señalan que la cocaína no es una droga nueva y que es una droga peligrosa que compromete la salud individual y social. En México no se le consideró un problema sino hasta la década de 1980. En la siguiente década, con el cierre de la ruta del Caribe para sacar la droga de la región andina hacia Estados Unidos y Europa, la cocaína encuentra su camino por el corredor América Central-México y su consumo se extiende en nuestro país, aumentando 
entre los años 1988 y 2011 casi ocho veces el número de personas que habían experimentado con ella.

En los usuarios de drogas que solicitaron tratamiento en CIJ, en la década de 1990 el consumo de cocaína mostró un marcado incremento, con lo que pasó de una de cada diez personas a siete de cada diez para el año 2000. Luego se mantuvo estable hasta 2007, y posteriormente inició un periodo de decremento con menos de cinco usuarios por cada diez personas que buscan ayuda. Esta tendencia incluye el crack y se observa también en la población atendida en los centros no gubernamentales y entre estudiantes de la Ciudad de México, donde se cuenta con tres décadas y media de mediciones periódicas.

Estos datos muestran que la percepción de la peligrosidad de la droga ha tenido variaciones a lo largo del tiempo, como también ha variado el consumo, con periodos de crecimiento y decremento a partir de que se inician los estudios epidemiológicos. Estas tendencias, producto de variaciones en los mercados externos, a factores internos y a las políticas públicas, deben ser consideradas al evaluar programas de prevención para anticipar la demanda de tratamiento.

La cocaína sigue considerándose como una droga peligrosa, factor que favorece los programas de prevención que no requieren invertir recursos para lograr que se le considere como tal, a diferencia del alcohol, y aplicar los recursos en otras fases del problema.

El hecho de que se use con menos frecuencia en forma inyectada redunda en menos riesgo de diseminación de enfermedades infecciosas. Sin embargo, esta forma de uso debe monitorearse cuidadosamente por los riesgos asociados de transmisión de otras enfermedades como VIH y hepatitis C.

La observación de que una cuarta parte de los usuarios la consuma fumada, como crack o "piedra", y que sean sobre todo personas desempleadas que además usan en mayor proporción otras drogas que quienes recurren a su forma en polvo, obliga a instaurar programas de identificación y atención de esta población, que presenta más carencias y menor rezago en la atención. La asociación entre esta forma de uso y la violencia en sus tres formas descritas en el documento (efecto farmacológico, robo para conseguir dinero y participación en narcomenudeo para obtener la droga) es importante, por lo que los programas de atención deben incluir acciones de desarrollo social, de prevención del delito y oportunidades de desarrollo emocional y social para estos grupos.

Los sistemas de vigilancia epidemiológica deben estar atentos al reporte del consumo de pasta base, pues ésta es altamente peligrosa por su bajo precio y rapidez de absorción, que lleva a las personas con dependencia a un uso repetido y un rápido deterioro, a fin de enfrentar sus retos.

La cocaína sigue siendo una droga consumida en su mayoría por hombres; aun así, entre las mujeres que la han consumido, una mayor proporción prefiere la forma fumada.

Esta observación, junto con los riesgos que conlleva el consumo durante el embarazo, indica la necesidad de iden- tificar y dar atención a las mujeres embarazadas y en riesgo de embarazo. Sus estilos de vida, con frecuente exposición a la violencia, las ponen en especial riesgo de tener un embarazo no deseado, y su enfermedad a la exposición de su bebé a la droga in utero y después del parto.

El hecho de que una tercera parte de los usuarios de cocaína empiece a consumirla antes de los 18 años y que haber fumado o consumido alcohol en la adolescencia temprana incremente la probabilidad de experimentación con cocaína indican los caminos hacia la prevención con programas integrados que permitan prevenir el uso de tabaco, retrasar la edad de inicio del consumo de alcohol y reducir la exposición de los adolescentes a la cocaína, al tiempo que se les dota de las habilidades para tener un desarrollo alterno al uso de drogas y con programas de desarrollo social que atienda las necesidades de los grupos en mayor rezago que consumen las formas más dañinas de esta droga. La observación de que su uso se extiende después de la adolescencia obliga a mantener por más tiempo los programas adaptados a las necesidades de personas que pasan por otros ciclos de vida.

$\mathrm{Su}$ asociación con las diferentes formas de violencia descritas en el documento obliga a poner énfasis en la diferenciación de estos fenómenos, ya que, a pesar de que pueden tener en común la manifestación de una conducta violenta o ser víctima de delito o agresión, tienen una etiología diferente y por tanto también deben de ser diferentes los programas cuya meta es su control y disminución.

El documento explica los mecanismos mediante los cuales la droga produce placer, factor que es lo que lleva a los usuarios a buscarla, pero también señala el proceso de la dependencia, especialmente con variaciones de corta duración, las razones que subyacen a las recaídas tan frecuentes en la población de personas con dependencia grave en tratamiento y de los daños producidos en múltiples órganos y en la vida social.

Los múltiples efectos sobre la salud de los usuarios, descritos en este trabajo, señalan la necesidad de que el tratamiento, además de integrar la atención a problemas derivados del consumo de alcohol, tabaco y otras drogas, siga un modelo integral que permita atender la salud física, mental y social de los usuarios.

El estudio de los adictos en tratamiento ha permitido conocer su comorbilidad, especialmente con la depresión, los trastornos de conducta y los problemas familiares y sociales que presentan, incluida la conducta delictiva.

El conocimiento generado por la investigación ha permitido avanzar en las opciones farmacológicas y psicoterapéuticas. Así, los modelos de intervención más útiles han sido los que se basan en el conocimiento de la adicción y su desarrollo. El conocimiento generado también permite diseñar tratamientos ad hoc a las necesidades en la salud física, mental y social de cada persona con dependencia, lo que facilita la adherencia al tratamiento. De este modo, el reto es llevar este conocimiento a la comunidad y a los encargados 
de programas de prevención y tratamiento. Ante todo ello, la investigación tiene mucho más que decir en términos del desarrollo de medicamentos y de mejores modelos de intervención.

\section{REFERENCIAS}

1. Escohotado A. Historia de las drogas 2. Alianza Editorial, Madrid 1997.

2. Freud S. Escritos sobre la cocaína. $1^{\text {a }}$ Ed. Editorial Anagrama. Colección Argumentos, Barcelona, 1980.

3. Goldstein RA, DesLauriers C, Burda AM. Cocaine: history, social implications, and toxicity: a review. Dis Mon. 2009 Jan; 55(1):6-38.

4. Tapia-Conyer R, Cravioto, De la Rosa B, Galván F. Historia natural del consumo de la cocaína: el caso de Ciudad Juárez, Chihuahua. Salud Mental 2003; 26(2):12-21.

5. The World Bank, World Development Report, Development and the next generation. The International Bank for Reconstruction and Development, Washington D.C., 2007.

6. Convención Única de 1961 sobre estupefacientes, enmendada por el Protocolo que modifica la Convención única de 1961 sobre estupefacientes. («BOE núm. 264/1981, de 4 de noviembre de 1981»). Nueva York, 8 de agosto de 1975.

7. Koob JF, Le Moal M. Neurobiology of addiction. Academic Press, San Diego, 2006.

8. Sthal SM. Sthal's essential psychopharmacology. 3a. edición. Cambridge University Press, New York, 2008.

9. Méndez Díaz M, Ruiz Contreras AE, Prieto Gómez B, Romano A, Caynas S, Prospéro García O. El cerebro y las drogas, sus mecanismos neurobiológicos. Salud Mental 2010; 33:451-456.

10. Nade MA, Czoty PW. PET imaging of dopamine D2 receptors in monkeys models of cocaine abuse: genetic predisposition versus environmental modulation. Am. J. Psychiat 2005; 162:1473-1482.

11. Kalivas PW, Pierce RCh, Sorg BA. Papel de la sensibilización en la paranoia y las psicosis inducidas por psicoestimulantes. En; Palomo T, Beninger RJ, Jiménez-Arriero MA, Archer T, eds. Trastornos esquizopsicóticos. Madrid, Síntesis, 2000; 85-93.

12. Aron AR, Robbins TW, Poldrack RA (2004) Inhibition and the right inferior frontal cortex. Trends Cogn Sci 8:170-177.

13. Hong S, Jhou TC, Smith M, Saleem KS, Hikosaka O. Negative reward signals from lateral habenula to dopamine neurons are mediated by rostromedial tegmental nucleus in primates. The Journal of Neuroscience, 2011; 31:11457-11471.

14. Centro Nacional para la Prevención y Control de las Adicciones. Comisión Nacional contra las Adicciones. Instituto Nacional de Psiquiatría Ramón de la Fuente. Instituto Nacional de Salud Pública. Secretaría de Salud Encuesta Nacional de Adicciones 2011. Secretaría de Salud, 2012. México, D.F.

15. Medina-Mora ME, Sepúlveda J, Rascón ML, Lazcano F, Mariño MC, Tapia CR, Otero MR, Solache G, Villatoro J, López EK. Patrones de consumo de alcohol y síntomas de dependencia en una región del centro de la República Mexicana. Rev. Latinoamericana sobre Alcohol y Drogas 1989; 1(1):47-56.

16. SS, CONADIC, INPRFM, INSP. (2009). Encuesta Nacional de Adicciones 2008. Secretaria de Salud, México.

17. Ortiz A, Martínez R, Meza D. Grupo Interinstitucional para el desarrollo del Sistema de Reporte de Información en Drogas. Resultados de la Aplicación de la Cédula: "Informe Individual sobre Consumo de Drogas". Tendencias en el área metropolitana No. 49, Noviembre de 2010. Ed. Instituto Nacional de Psiquiatría Ramón de la Fuente Muñiz. México, D.F.

18. Villatoro J, Gaytán E, Moreno M, Gutiérrez M, Oliva N, et al. (2010) Consumo de Alcohol, Tabaco y otras Drogas en la Ciudad de México.
Medición 2009. Instituto Nacional de Psiquiatría Ramón de la Fuente Muñiz. México, D.F.

19. García R, Fukushima E, Gracia S, Jiménez K, Cielo B. Prueba de instrumentos para la evaluación de un programa de tratamiento para usuarios de estimulantes. Centros de Integración Juvenil, Dirección de Investigación y Enseñanza, Subdirección de Investigación, Informe de investigación 06-12, México, 2006.

20. Gutiérrez López AD. Reporte del Sistema de Información Epidemiológica del Consumo de Drogas, Segundo semestre de 2010, Centros de Integración Juvenil, Dirección de Investigación y Enseñanza, Subdirección de Investigación, Informe de Investigación 11-07a, México, 2011.

21. García AR, Díaz-Guerrero R, Reyes LI, Medina-Mora ME, Andrade PP, Reidl ML. Indicadores psicosociales de motivación del consumo de marihuana y/o cocaína. Adiciones 2006; 18(4):387-398.

22. Jiménez K, García R, Balanzario C. Consumo de estimulantes y su relación con la violencia, Centros de Integración Juvenil, Dirección de Investigación y Enseñanza, Subdirección de Investigación, Informe de Investigación 06-13, México, 2006.

23. Cole C, Jones L, McVeigh J, Kicman A, Syed Q, Bellis M.. Adulterants in illicit drugs: a review of empirical evidence. Drug Test Anal. 2011 Feb; 3(2):89-96.

24. Fajemirokun-Odudeyi O, Lindow S. Obstetric implications of cocaine use in pregnancy: a literatura review. European Journal of Obstetrics and Gynecology and Reproductive Biology. 2004; 112:2-8.

25. Sharp C, Baker P, Goulder C, Ramsay M, Sondhi A. Drug misuse declared in 2000: key results from British Crime Survey. Findings 2001. Vol 149. London: Home Office.

26. Little B, Snell L, Palmore M, Gilstrap L. Cocaine use in pregnant women in a large public hospital. Am J Perinatol 1988; 5:206-207.

27. Chasnoff I, Landress $H$, Barrett $M$. The prevalence of illicit-drug or alcohol use during pregnancy and discrepancies in mandatory reporting in Pinellas County, Florida. N Engl J Med 1990; 322:1202-1206.

28. Behnke M, Eyler F, Conlon M, Woods N, Casanova O. Multiple risk factors do not identify cocaine use in rural obstetrical patients. Neurotoxicol Teratol 1994; 16:479-484.

29. Minnes S, Lang A, Singer L. Prenatal Tobacco, Marijuana, Stimulant and opiate exposure: outcomes and Practice implications. Adiction Science and Clinical Practice. 2011; 6:57-70.

30. Dawes M, Chowienczyk P. Pharmacokinetics in pregnancy. Best Practice and Research. 2001; 15(6):819-826.

31. Chasnoff I. Drug use in pregnancy. 1988. Kluwer Academic Publishers. UK.

32. Chasnoff I, Lewis D. Cocaine metabolism during pregnancy. Pediatr Res 1988; 23:257A.

33. Schenker S, Yang Y, Johnson RF et al. The transfer of cocaine and its metabolites across the term placenta. Clin Pharmacol Ther 1993; 53(3):329-339.

34. Chokshi S, Gal D, Isner J. Evidence that fetal distress in newborns of cocaine user is due to vascular spasm and may be attenuated to pretreatment with Diltiazem [abstract]. Circulation 1989; 80 (Suppl II): 185.

35. Acker D, Sachs B, Tracey K, Wise W. Abruptio placentae associated with cocaine use. Am J Obstet Gynecol 1983; 146:220-221.

36. Dombrowski M, Wolfe $H$, Welch $R$, Evans M. Cocaine abuse is associated with abruptio placentae and decreased birth weight but not shorter labor. Obstet Gynecol 1991; 77:139-141.

37. Addis A, Moretti M, Ahmed Syed F, Einarson TR, Koren G. Fetal effects of cocaine: An updated meta-analysis. Reproductive Toxicology 2001 15(4):341-369.

38. Morgan M, Honnebier M, Mecenas C, Nathanielsz P. Cocaine effect on plasma oxytocin concentrations in the baboon during late pregnancy. Am J Obstet Gynecol 1996; 174:1026-1027.

39. Chasnoff I, Griffith D, MacGregor S, Dirkes K, Burns K. Temporal patterns of cocaine use in pregnancy: perinatal outcome. JAMA 1989; 261:1741-1744. 
40. Bingol N, Fuchs M, Diaz V, Stone R, Gromisch D. Teratogenicity of cocaine in humans. J Pediatr 1987; 110:93-96.

41. Jhaveri M, Schechter C, Gertner M, Holzman I. Perinatal cocaine/crack exposure in infants: a different perspective. Neonatal Intens Care 1993; May-June:18-20.

42. Singer LT, Arendt R, Minnes S, Farkas K, Salvator A. Neurobehavioral outcomes of cocaine-exposed infants. Neurotoxicology and Teratology 2000; 22(5):653-666.

43. Lee CT, Chen J, Hayashi T, Tsai SY, Sanchez J, et al. A mechanism for the inhibition of neural progenitor cell proliferation by cocaine. PLoS Medicine 2008; 5(6):e117 doi:10.1371/journal.pmed.0050117.

44. Richardson GA, Goldschmidt L, Willford J. The effects of prenatal cocaine use on infant development. Neurotoxicology and Teratology 2008 30(2):96-106.

45. Carmody D, Bennet D, Lewis M. The effects of prenatal cocaine exposure and gender on inhibitory control and attention. Neurotoxicology and Teratology 2011; 33(2):61-68.

46. Lewis BA, Minnes S, Short EJ, Weishampel P, Satayathum S, Min MO, Nelson S, Singer LT. The effects of prenatal cocaine on language development at 10 years of age. Neurotoxicology and Teratology 2011; 33(1):17-24.

47. Richardson GA, Goldschmidt L, Larkby C. Effects of prenatal exposure cocaine on growth: a longitudinal analysis. Pediatrics 2007 120(4):e1017.

48. Arsenault L, Cannon M, Poulton R, Murray R, Caspi A, Moffit TE. Cocaine use in adolescent and risk for adult psychosis: longitudinal prospective study. Br MedJ2002; 325:1212-1213.
49. Kalivas PW, Pierce RCh, Sorg BA. Papel de la sensibilización en la paranoia y las psicosis inducidas por psicoestimulantes. En; Palomo T, Beninger RJ, Jiménez-Arriero MA, Archer T, eds. Trastornos esquizopsicóticos. Madrid, Síntesis, 2000; 85-93.

50. Zammit S, Allebeck P, Andreasson S, Lundberg I, Lewis G. Self reported cocaine use as a risk factor for schizophrenia in Swedish conscripts of 1969: historical cohort study. Br Med J 2002; 325:1199-1201.

51. Gearon JS, Bellak AS. Sex differences in illness presentation, course and level of functioning in substance-abusing schizophrenia patients. Schizophr Res 2000; 43:65-70.

52. Valderrama JC, Cervera G, de Vicente P, Bolinches F, Ochando B, Sánchez $P$. Tratamiento de los trastornos de la personalidad en sujetos con trastorno por uso de sustancias. En: Rubio G, López-Muñoz F, Álamo C, Santo-Domingo J, eds. Trastornos psiquiátricos y abuso de sustancias. Madrid, Médica Panamericana, 2002; 357-384.

53. Levin FR, Evans SM, Kleber HD. Prevalence of adult attention-deficit hyperactivity disorder among cocaine abusers seeking treatment. Drug Alcohol Depend 1998; 52:15-25.

54. Weiner NA \& Wolfgang. Pathways to criminal violence. 1989. SAGE Focus Editions, Michigan.

55. Ghodse H. International drug control into the $21^{\text {st }}$ century. 2008. Ashgate Publishing Limited, United Kingdom.

56. Valdez A, Kaplan CH, Curtis R. Aggressive crime, alcohol and drug use and concentrate poverty in 25 U.S. urban areas. Am Journal of drug and alcohol abuse 2007; 33:595-603.

Artículo sin conflicto de intereses 